\title{
Differential Role of Muscarinic Transmission within the Entorhinal Cortex and Basolateral Amygdala in the Processing of Irrelevant Stimuli
}

\author{
Segev Barak ${ }^{1,2}$ and Ina Weiner*,I \\ 'Department of Psychology, Tel-Aviv University, Tel-Aviv, Israel
}

\begin{abstract}
Cholinergic projections to the entorhinal cortex (EC) and basolateral amygdala (BLA) mediate distinct cognitive processes through muscarinic acetylcholine receptors (mAChRs). In this study, we sought to further differentiate the role of muscarinic transmission in these regions in cognition, using the latent inhibition ( $\mathrm{LI}$ ) phenomenon. $\mathrm{LI}$ is a cross-species phenomenon manifested as poorer conditioning to a stimulus experienced as irrelevant during an earlier stage of repeated non-reinforced pre-exposure to that stimulus, and is considered to index the ability to ignore, or to in-attend to, irrelevant stimuli. Given our recent findings that systemic administration of the mAChR antagonist scopolamine can produce two contrasting $\mathrm{LI}$ abnormalities in rats, ie, abolish LI under conditions yielding $\mathrm{LI}$ in non-treated controls, or produce abnormally persistent $\mathrm{LI}$ under conditions preventing its expression in non-treated controls, we tested whether $m A C h R$ blockade in the EC and BLA would induce LI abolition and persistence, respectively. We found that intra-EC scopolamine infusion ( $\mathrm{I}, 10 \mu \mathrm{g}$ per hemisphere) abolished $\mathrm{LI}$ when infused in pre-exposure or both pre-exposure and conditioning, but not in conditioning alone, whereas intra-BLA scopolamine infusion led to persistent $\mathrm{LI}$ when infused in conditioning or both stages, but not in pre-exposure alone. Although cholinergic innervation of the EC and BLA has long been implicated in attention to novel stimuli and in processing of motivationally significant stimuli, respectively, our results provide evidence that EC mAChRs also have a role in the development of inattention to stimuli, whereas BLA mAChRs have a role in re-attending to previously irrelevant stimuli that became motivationally relevant.
\end{abstract}

Neuropsychopharmacology (2010) 35, I073-1082; doi:I0.1038/npp.2009.210; published online I3 January 2010

Keywords: latent inhibition; attention; scopolamine; entorhinal cortex; basolateral amygdala; schizophrenia

\section{INTRODUCTION}

Cholinergic projections from the basal forebrain (BF) have a fundamental role in cognitive processes, and abnormalities of the cholinergic system are implicated in cognitive dysfunctions associated with several neurological/neuropsychiatric disorders (for reviews, see Everitt and Robbins, 1997; Fibiger, 1991; Hasselmo, 2006; Power et al, 2003; Sarter et al, 2003). Blockade of muscarinic acetylcholine receptors (mAChRs) has been shown to induce memory and attentional deficits in both animals and humans (Blokland, 1995; Ebert and Kirch, 1998; Everitt and Robbins, 1997; Green et al, 2005). Two of the cholinergic projections from the $\mathrm{BF}$, those to the entorhinal cortex (EC) and the

\footnotetext{
*Correspondence: Professor I Weiner, Department of Psychology, Tel-Aviv University, Ramat-Aviv 39040, Tel-Aviv 69978, Israel, Tel: + 9723640 8993, Fax: +972 3640 9547, E-mail: weiner@post.tau.ac.il ${ }^{2}$ Current address: Ernest Gallo Clinic and Research Center, University of California, San Francisco 5858 Horton St., Suite 200, Emeryville, CA 94608, USA, E-mail: sbarak@gallo.ucsf.edu

Received 18 August 2009; revised 30 November 2009; accepted 30 November 2009
}

basolateral amygdala (BLA), have been shown to mediate distinct cognitive processes through mAChRs (Hasselmo, 2006; Hasselmo and McGaughy, 2004; Ingles et al, 1993; Power, 2004). This study sought to further differentiate the role of muscarinic transmission in these two regions in cognition, using the latent inhibition (LI) phenomenon.

LI is one of the best documented cross-species manifestations of attentional selectivity in associative learning (Lubow, 1989; Lubow and Weiner, 2009). It is manifested when repeated non-reinforced pre-exposure to the to-beconditioned stimulus reduces the efficacy of this stimulus to acquire and/or express conditioned responses when paired with reinforcement. We have recently shown that systemic administration of the mAChR antagonist scopolamine in rats can produce two contrasting LI abnormalities as a function of dose and stage of administration. At low doses, scopolamine abolishes LI, whereas rats treated with higher doses persist in expressing LI under conditions that prevent/ reduce LI expression in untreated rats. Scopolamineinduced LI abolition is due to the action of the drug in the pre-exposure stage (Barak and Weiner, 2007), whereas scopolamine-induced LI persistence is due to its action in 
the conditioning stage (Barak and Weiner, 2009). Thus, scopolamine produces contrast poles of impairment in attentional selectivity: at low doses it impairs the capacity to inattend to irrelevant stimuli, whereas at a higher dose it impairs the capacity to re-attend to irrelevant stimuli when they become relevant through pairings with reinforcement. By extension, these findings suggest that normal muscarinic transmission is required for the acquisition of inattention to irrelevant stimuli as well as for the adjustment of responding based on changes in the relevance of stimuli (Barak, 2009).

It has been previously shown that lesions to the EC and BLA produce LI abolition and persistence, respectively (Coutureau et al, 1999, 2000; Schiller and Weiner, 2004, 2005; Schiller et al, 2006; Shohamy et al, 2000; Yee et al, 1995) (but see Coutureau et al, 2001). Moreover, reversible EC inactivation, similar to our systemic scopolamine injections, were effective in pre-exposure but not conditioning (Jeanblanc et al, 2004; Lewis and Gould, 2007a; Seillier et al, 2007). No stage selectivity has been tested for BLA lesions. In this study, we tested the hypothesis that LI abolition and persistence produced by EC and BLA lesions are mediated by impaired muscarinic transmission in these regions. More specifically, we expected two outcomes. First, that blockade of muscarinic transmission in the EC and BLA will result in LI loss and persistence, respectively. Second, that these effects will show stage specificity, namely, that muscarinic blockade within the EC will abolish LI by action in pre-exposure, whereas muscarinic blockade within the BLA will produce persistent LI by action in conditioning. For this aim, we tested the effects of scopolamine infusion into EC or BLA before pre-exposure and/or before conditioning using experimental protocols that produce LI or prevent its expression in control rats, therefore allowing demonstration of LI abolition or persistence, respectively, in treated rats.

\section{MATERIALS AND METHODS}

\section{Subjects}

Male Wistar rats (Tel Aviv University Medical School, Tel Aviv, Israel), approximately 3 months old and weighing $300-460 \mathrm{~g}$ at the time of surgery, were housed individually under reversed cycle lighting (lights on: 1900-0700 h) with ad lib food and water except for the duration of the LI experiments (see below). All experimental protocols conformed to the guidelines of the Institutional Animal Care and Use Committee of Tel Aviv University, Israel, and to the guidelines of the NIH (animal welfare assurance number A5010-01, expires on 30/09/2011). All efforts were made to minimize the number of animals used and their suffering.

\section{Surgery}

Rats were given an i.p. injection of diazepam $(0.6 \mathrm{mg} / \mathrm{kg})$ and $5 \mathrm{~min}$ later were anesthetized with an i.p. injection of avertin $(10 \mathrm{ml} / \mathrm{kg})$. They were placed in a stereotaxic frame (David Kopf Instruments, Tujunga, CA), and an incision was made into the scalp to expose the skull. The vertical coordinates of bregma and lambda were measured in order to align them in the same (level head) plane. Holes were drilled and rats were implanted bilaterally with 23-gauge indwelling stainless steel guide cannulae (Plastic Ones, USA) aimed $1 \mathrm{~mm}$ above the EC or the BLA according to the following coordinates, measured relative to the skull at bregma (Paxinos and Watson, 1998): EC: $7.1 \mathrm{~mm}$ posterior to bregma, $5 \mathrm{~mm}$ lateral to the midline, $8.0 \mathrm{~mm}$ ventral to the skull; BLA: $2.8 \mathrm{~mm}$ posterior to bregma, $5 \mathrm{~mm}$ lateral to the midline, $8.5 \mathrm{~mm}$ ventral to the skull. Thus, the guide cannulae were aimed just dorsal to the main target area. The cannulae were anchored to the skull with three stainless steel screws and dental cement. Stainless steel stylets (27G) cut to extend $1.0 \mathrm{~mm}$ beyond the tip of the guide cannulae were placed into the cannulae to prevent occlusion. After surgery, rats were returned to their home cages and allowed 7-12 days of recovery before the initiation of behavioral testing. During recovery, rats were given daily health checks in which the experimenter weighted them and replaced missing stylets.

\section{Behavioral Apparatus and Procedure}

LI was measured in a thirst-motivated conditioned emotional response (CER) procedure by comparing the suppression of drinking to a tone previously paired with a footshock in rats that received non-reinforced exposure to the tone before conditioning (pre-exposed, $\mathrm{PE})$ and in rats for whom the tone was novel (non-pre-exposed, NPE).

Rats were tested in Campden Instruments rodent test chambers (model 410) with a retractable bottle. When the bottle was not present, the hole was covered by a metal lid. Licks were detected by a Campden Instruments drinkometer (model 435). The PE to-be-conditioned stimulus was a $10 \mathrm{~s}, 80 \mathrm{~dB}, 2.8 \mathrm{kHz}$ tone produced by a Sonalert module (model SC 628). Shock was supplied through the floor by a Campden Instruments shock generator (model 521/C) and shock scrambler (model 521/S) set at $0.5 \mathrm{~mA}$ and $1 \mathrm{~s}$ duration. Equipment programming and data recording were computer controlled.

Before surgery, rats were put on a $23 \mathrm{~h}$ water restriction schedule for 8 days. They were handled for about $2 \mathrm{~min}$ daily on the first 5 days and trained to drink in the experimental chamber for 15 min daily for the next 3 days. Water in the test apparatus was given in addition to the daily ration of $1 \mathrm{~h}$ given in the home cages. After recovery from surgery, water restriction schedule was re-instated. Rats were handled daily for 3 days, and on the next 3 days, re-trained to drink in the experimental chamber for $15 \mathrm{~min} /$ day. On the next 4 days, the LI procedure was conducted, consisting of the following stages:

Pre-exposure. With the bottle removed, the PE rats received 40 tone presentations with an inter-stimulus interval of $40 \mathrm{~s}$. The NPE rats were confined to the chamber for an identical period of time without receiving the tone.

Conditioning. With the bottle removed, rats received 2 (weak conditioning; Experiments 1-3) or 5 (strong conditioning; Experiments 3 and 4) tone-shock pairings given $5 \mathrm{~min}$ apart. Shock immediately followed tone termination. The first tone-shock pairing was given $5 \mathrm{~min}$ after the start of the session. After the last pairing, rats were left in the experimental chamber for an additional $5 \mathrm{~min}$. 
The two (weak and strong) conditioning protocols are routinely used by us to demonstrate LI abolition and persistence in the present CER procedure. Specifically, weak conditioning produces LI in non-treated controls and thus allows the demonstration of treatment-induced LI abolition. Conversely, strong conditioning prevents LI in non-treated controls and thus allows the demonstration of treatmentinduced abnormally persistent LI.

Rebaseline. Rats were given a 15 min drinking session as in initial training. Data of rats that failed to complete 600 licks were dropped from the analysis.

Test. Each rat was placed in the chamber and allowed to drink from the bottle. When the rat completed 75 licks, the tone was presented for $5 \mathrm{~min}$. The following times were recorded: time to first lick, time to complete licks 1-50, time to complete licks 51-75 (before tone onset), and time to complete licks 76-100 (after tone onset). Times to complete licks 76-100 were submitted to logarithmic transformation to allow parametric analysis of variance. Longer log times indicate stronger suppression of drinking. LI is defined as significantly shorter log times to complete licks 76-100 of the PE compared with NPE rats.

\section{Drugs and Infusions}

Rats received bilateral infusions of either physiological saline $(0.9 \%$ sodium chloride, $\mathrm{pH} 7.0)$ or scopolamine hydrobromide ( 1 or $10 \mu \mathrm{g} / 0.5 \mu \mathrm{l}$ in physiological saline; Sigma, Israel). The doses of scopolamine were based on similar behavioral studies that used scopolamine infusion into cortical and/or hippocampal formation regions (Barros et al, 2001; Herremans et al, 1996; Warburton et al, 2003; Winters et al, 2006). Importantly, these doses were lower than the doses shown before to impair classical conditioning when infused to BLA (See et al, 2003), because impairment of conditioning per se (impaired conditioning in the NPE groups) masks the effects of experimental manipulations on LI. Infusion volume was based on a routinely used volume in similar behavioral studies ((Barros et al, 2001; Lewis and Gould, 2007a, b; Roesler et al, 2002; Seillier et al, 2007) for EC; (Barros et al, 2001; Chhatwal et al, 2009; Milton et al, 2008; See et al, 2001, 2003; Zhao et al, 2009) for BLA). Infusions were given in a different room from the behavioral testing area. Animals were gently restrained by the experimenter throughout the infusion process. Stylets were removed, and the stainless steel 28-gauge injection cannulae, which extended $1.00 \mathrm{~mm}$ beyond the tip of the guide cannulae, were inserted into the guide cannulae. The injection cannulae were attached to tubing attached to $5 \mu \mathrm{l}$ microsyringes mounted on a motorized syringe pump (model WPI SP200). Bilateral infusions were performed simultaneously. A total of $0.5 \mu \mathrm{l}$ per hemisphere was delivered over $120 \mathrm{~s}$. After the pump was shut off, injection cannulae were kept in place for additional $90 \mathrm{~s}$ to allow for diffusion of the infusate. Injectors were then removed, stylets were replaced, and animals were placed into the test chambers. On the last day of handling and the second day of drinking training (see above in section Behavioral Apparatus and Procedure), rats experienced a 'mock' infusion identical in all respects to the procedure described above except that the injection cannulae contained no liquid and the tubes were unconnected to the pump. This was done to habituate the animals to the general protocol of the infusion procedure, including insertion of the infusion cannulae and the sound of the pump.

The EC and BLA infusions were given before the preexposure and/or the conditioning stages depending on the aim of the experiment (see Table 1).

\section{Histology}

After the completion of behavioral testing, rats were anesthetized with an overdose of pentobarbital $(60 \mathrm{mg} / \mathrm{ml})$ and perfused intracardially with physiological saline, followed by $10 \%$ formalin. Their brains were removed from the skulls and stored in $10 \%$ formalin for at least $24 \mathrm{~h}$, and then in $30 \%$ sucrose solution for at least $24 \mathrm{~h}$ before being sectioned in the coronal plane at $40 \mu \mathrm{m}$ thickness. Sections were mounted on a gelatinized glass slide and stained with thionin blue for histological examination. Verification of cannulae placements used the atlas of Paxinos and Watson (1998).

\section{Data Analysis}

Times to complete licks 51-75 and mean log times to complete licks 76-100 were analyzed using a two- or threeway ANOVAs with main factors of pre-exposure, intracerebral treatment (Experiments 1-4), and number of toneshock pairings (Experiment 3). LSD post hoc comparisons were used to assess the difference between the PE and NPE groups within each treatment condition.

\section{Experimental Design}

Experiments 1 and 2 tested the effects of intra-EC scopolamine infusion on LI, and Experiments 3 and 4 tested the effects of intra-BLA scopolamine infusion on LI. The region- and stage-specific effects of intra-EC and intraBLA scopolamine infusion were determined by combining weak and/or strong conditioning protocols (see Behavioral Apparatus and Procedure section) and drug infusion in the pre-exposure and/or conditioning stage (see Table 1 and below).

\section{RESULTS}

\section{Histological Assessment}

A schematic reconstruction of cannulae placement in the EC and BLA is presented in the Supplementary Figure S1a and $b$, respectively. Data from rats in which cannulae tips were located outside the EC (two in Experiment 1; two in Experiment 2) or the BLA (one in Experiment 3; three in Experiment 4) were excluded from the statistical analysis.

\section{Behavior}

Experiment 1 -Effects of intra-EC scopolamine infusion (1 or $10 \mu \mathrm{g}$ per hemisphere) before pre-exposure on LI with weak conditioning. As systemic scopolamine
. 
Table I Experimental Design of the Five Experiments

\begin{tabular}{|c|c|c|c|c|c|}
\hline & \multirow{2}{*}{ Tone-shock pairings } & \multirow{2}{*}{ Treatment group } & \multicolumn{2}{|c|}{ Infusion } & \multirow{2}{*}{ Results } \\
\hline & & & Pre-exposure & Conditioning & \\
\hline \multirow[t]{2}{*}{ Experiment I-EC } & 2 & Scop in prex & Scop I $\mu g$ & Vehicle & No LI \\
\hline & & Scop in prex & Scop $10 \mu g$ & Vehicle & No LI \\
\hline \multirow[t]{3}{*}{ Experiment 2-EC } & 2 & Scop in cond & Vehicle & Scop $10 \mu g$ & LI \\
\hline & & Scop in both stages & Scop $10 \mu g$ & Scop $10 \mu g$ & No LI \\
\hline & 2 & Control & Vehicle & Vehicle & $\mathrm{LI}$ \\
\hline \multirow[t]{2}{*}{ Experiment 3-BLA } & & Scop in both stages & Scop $10 \mu g$ & Scop $10 \mu g$ & $\mathrm{LI}$ \\
\hline & & Control & Vehicle & Vehicle & No LI \\
\hline \multirow[t]{2}{*}{ Experiment 4-BLA } & 5 & Scop in prex & Scop $10 \mu g$ & Vehicle & No LI \\
\hline & & Scop in cond & Vehicle & Scop $10 \mu g$ & $\mathrm{LI}$ \\
\hline
\end{tabular}

Abbreviations: BLA, basolateral amygdala; EC, entorhinal cortex; LI, latent inhibition; prex, pre-exposure; scop, scopolamine; cond, conditioning.

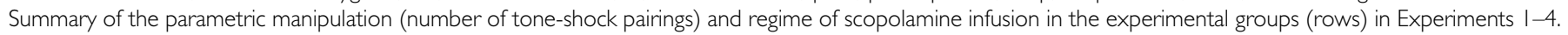

abolishes LI when administered in pre-exposure but not in conditioning (Barak and Weiner, 2007), and there is extensive evidence that inactivation of the EC during pre-exposure abolishes LI (Jeanblanc et al, 2004; Lewis and Gould, 2007a; Seillier et al, 2007), we had a strong prediction that intra-EC scopolamine infusion before pre-exposure would abolish LI. Therefore, here we confined the infusion to the pre-exposure stage. Weak conditioning that leads to LI in non-treated controls and allows demonstration of LI abolition was used.

The experiment included six experimental groups ( $n$ per group $=5-6)$ in a $2 \times 3$ design with main factors of pre-exposure (NPE, PE) and intra-EC treatment (vehicle, $1,10 \mu \mathrm{g}$ scopolamine). The six experimental groups did not differ in their times to complete licks 51-75 before tone onset (all $p$-values $>0.05$; overall mean A period $=9.10 \mathrm{~s}$ ). Figure 1 presents the mean log times to complete licks 76-100 (after tone onset) of the six experimental groups. As can be seen, LI was present in vehicle-infused rats, but was dose-dependently abolished by intra-EC infusion of scopolamine. ANOVA yielded main effects of pre-exposure $\left(\mathrm{F}_{(1,26)}=6.57, p<0.02\right)$ and an interaction of pre-exposur$\mathrm{e} \times$ intra-EC treatment $\left(\mathrm{F}_{(2,26)}=5.858, p<0.01\right)$. Post hoc comparisons revealed a significant difference between the PE and NPE groups, ie, presence of LI in rats that received intra-EC infusion of vehicle $(p<0.001)$, but not in rats that received intra-EC infusion of 1 or $10 \mu \mathrm{g}$ scopolamine.

Experiment 2-Effects of intra-EC scopolamine infusion (10 $\mu \mathrm{g}$ per hemisphere) before conditioning or both stages on LI with weak conditioning. Having shown in Experiment 1 that LI was abolished, as predicted, by intra-EC scopolamine infusion in the pre-exposure stage, this

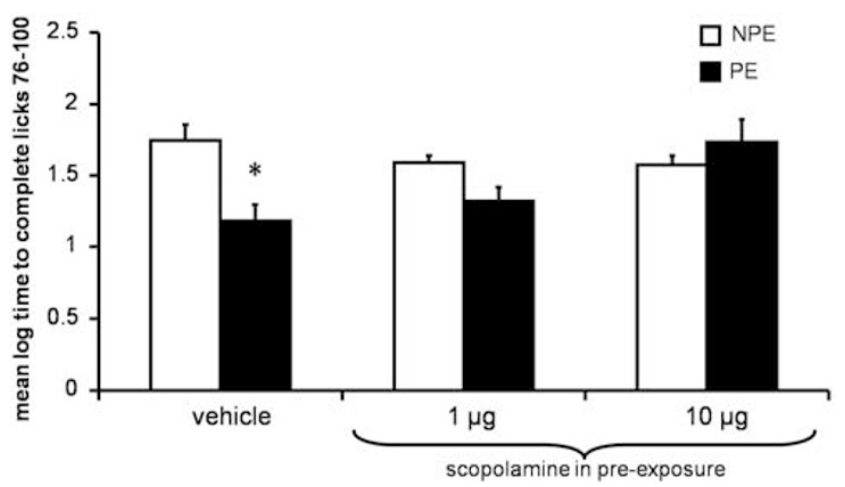

Figure I Effects of intra-entorhinal cortex scopolamine infusion (I or $10 \mu \mathrm{g}$ per hemisphere) before pre-exposure on LI with weak conditioning. Means and standard errors of the log times to complete licks 76-I00 (after tone onset) of the pre-exposed (PE) and non-pre-exposed (NPE) rats infused with vehicle or scopolamine $(1,10 \mu \mathrm{g})$ into the entorhinal cortex. Scopolamine was infused in the pre-exposure stage. Forty pre-exposures and two conditioning trials (weak conditioning) were used. Asterisk indicates a significant difference between the PE and NPE groups, namely, presence of $\mathrm{LI}$.

experiment sought to show that scopolamine will be ineffective if given only before conditioning, but will be effective if given before both pre-exposure and conditioning stages due to its action in pre-exposure. Weak conditioning was used to allow demonstration of LI abolition.

The experiment included six experimental groups ( $n$ per group $=6-7)$ in a $2 \times 3$ design with main factors of preexposure (NPE, PE) and intra-EC treatment (vehicle, $10 \mu \mathrm{g}$ scopolamine in conditioning, scopolamine in both stages). The six experimental groups did not differ in their times to 
complete licks $51-75$ before tone onset (all $p$-values $>0.05$; overall mean A period $=9.38 \mathrm{~s}$ ). Figure 2 presents the mean log times to complete licks 76-100 (after tone onset) of the six experimental groups. As can be seen, LI was present in vehicle-infused rats as well as in rats infused with scopolamine before conditioning, but not in rats that had scopolamine infused before both stages. ANOVA yielded main effects of pre-exposure $\left(\mathrm{F}_{(1,34)}=24.00, p<0.0001\right)$ and intra-EC treatment $\left(\mathrm{F}_{(1,34)}=4.14, p<0.025\right)$ and an interaction of pre-exposure $\times$ intra-EC treatment $\left(\mathrm{F}_{(2,34)}=7.57\right.$, $p<0.002)$. Post hoc comparisons confirmed presence of LI in rats that received intra-EC infusion of vehicle $(p<0.001)$ or scopolamine before conditioning $(p<0.0001)$, but not in rats that received intra-EC scopolamine infusion before both stages.

Experiment 3-Effects of intra-BLA scopolamine infusion (10 $\mu \mathrm{g}$ per hemisphere) before pre-exposure and conditioning on LI with weak and strong conditioning. Because we showed that BLA lesion leads to abnormally persistent LI (Schiller and Weiner, 2004, 2005; Schiller et al, 2006), we hypothesized that conditioning-based persistent LI produced by scopolamine is mediated by its effects in BLA. However, because some studies found that BLA perturbations disrupt LI (Coutureau et al, 2001; Schauz and Koch, 2000), we first tested whether scopolamine infusion would indeed produce persistent LI. For this, we used both the weak and the strong conditioning protocol to demonstrate that intra-BLA scopolamine spares LI under the former conditions, and causes abnormally persistent LI under the latter conditions. Scopolamine was infused in both preexposure and conditioning.

The experiment included eight experimental groups ( $n$ per group $=5-6)$ in a $2 \times 2 \times 2$ design with main factors of pre-exposure (NPE, PE), number of conditioning trials (two, five), and intra-BLA treatment (vehicle, $10 \mu \mathrm{g}$ scopolamine). The eight experimental groups did not differ in their times to complete licks 51-75 before tone onset (all $p$-values $>0.05$; overall mean A period $=7.93 \mathrm{~s}$ ). Figure 3

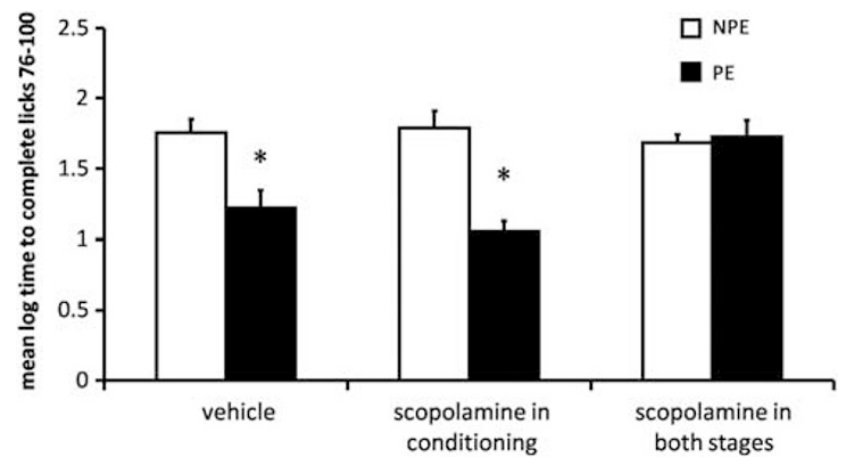

Figure 2 Effects of intra-entorhinal cortex scopolamine infusion ( $10 \mu \mathrm{g}$ per hemisphere) before conditioning or both stages on LI with weak conditioning. Means and standard errors of the log times to complete licks 76-100 (after tone onset) of the pre-exposed (PE) and non-pre-exposed (NPE) rats infused with vehicle or scopolamine $(10 \mu \mathrm{g})$ into the entorhinal cortex. Scopolamine was infused in the conditioning stage or in both preexposure and conditioning. Forty pre-exposures and two conditioning trials (weak conditioning) were used. Asterisk indicates a significant difference between the PE and NPE groups, namely, presence of LI. presents the mean log times to complete licks 76-100 (after tone onset) of the eight experimental groups. As can be seen, with two conditioning trials LI was present in both vehicle- and scopolamine-infused rats. In contrast, with five conditioning trials LI was absent in vehicle-infused rats, as expected under conditions of strong conditioning, but rats that received intra-BLA scopolamine infusion persisted in expressing LI. ANOVA yielded main effects of pre-exposure $\left(\mathrm{F}_{(1,39)}=37.50, p<0.0001\right)$, number of conditioning trials $\left(\mathrm{F}_{(1,39)}=5.97, p<0.02\right)$, and interactions of pre-exposur$\mathrm{e} \times$ number of conditioning trials $\left(\mathrm{F}_{(1,39)}=6.08, p<0.02\right)$ and pre-exposure $\times$ intra-BLA treatment $\left(\mathrm{F}_{(1,39)}=5.75\right.$, $p<0.025)$, but the pre-exposure $\times$ number of conditioning trials $\times$ intra-BLA treatment interaction did not reach significance $\left(\mathrm{F}_{(1,39)}=3.44, p=0.071\right)$. Post hoc comparisons confirmed presence of LI in vehicle- and scopolamineinfused rats that received two conditioning trials ( $p$-values $<0.0005$ ), as well as in scopolamine-infused rats that received five conditioning trials $(p<0.0005)$, but not in vehicle-infused rats that received five conditioning trials.

Experiment 4-Effects of intra-BLA scopolamine infusion (10 $\mu \mathrm{g}$ per hemisphere) before pre-exposure or conditioning on LI with strong conditioning. Having shown that scopolamine induces abnormally persistent LI when infused in both pre-exposure and conditioning, we tested at which of the stages this drug acts in the BLA to induce LI persistence. Given that systemic scopolamine induces LI persistence when administered in conditioning but not in pre-exposure (Barak and Weiner, 2009), we expected that intra-BLA scopolamine infusion would also induce LI persistence when infused immediately before conditioning. The strong conditioning protocol was used to allow demonstration of LI persistence.

The experiment included six experimental groups ( $n$ per group $=6-7)$ in a $2 \times 3$ design with main factors of preexposure (NPE, PE) and intra-BLA treatment (vehicle, $10 \mu \mathrm{g}$ scopolamine in pre-exposure, scopolamine in conditioning). The six experimental groups did not differ in their times to complete licks $51-75$ before tone onset (all $p$-values $>0.05$; overall mean A period $=10.32 \mathrm{~s}$ ). Figure 4 presents

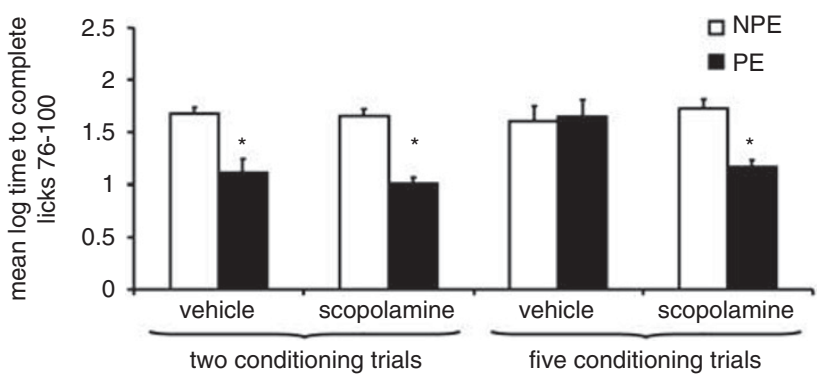

Figure 3 Effects of intra-basolateral amygdala scopolamine infusion ( I 0 g per hemisphere) on LI with weak or strong conditioning. Means and standard errors of the log times to complete licks 76-100 (after tone onset) of the pre-exposed (PE) and non-pre-exposed (NPE) rats infused with vehicle or scopolamine $(10 \mu \mathrm{g})$ into the basolateral amygdala that underwent conditioning with two (weak conditioning) or five (strong conditioning) trials. Forty pre-exposures were used. Asterisk indicates a significant difference between the PE and NPE groups, namely, presence of $\mathrm{LI}$. 


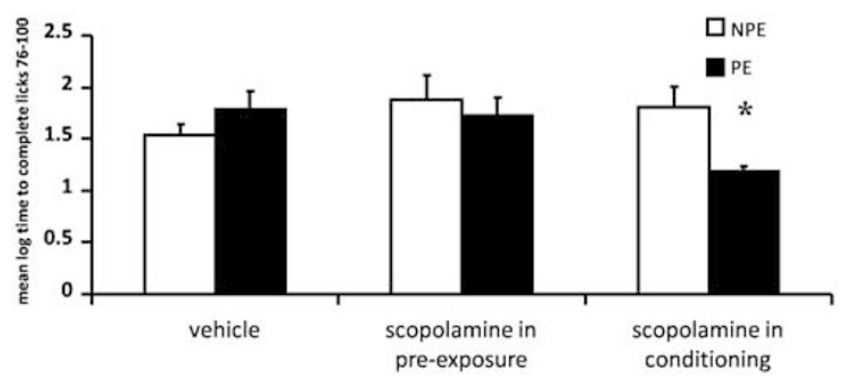

Figure 4 Effects of intra-basolateral amygdala scopolamine infusion ( $10 \mu \mathrm{g}$ per hemisphere) before pre-exposure or conditioning on $\mathrm{LI}$ with strong conditioning. Means and standard errors of the log times to complete licks 76-100 (after tone onset) of the pre-exposed (PE) and non-pre-exposed (NPE) rats infused with vehicle or scopolamine $(\mathrm{I} 0 \mu \mathrm{g})$ into the basolateral amygdala. Scopolamine was infused either in the pre-exposure stage or in the conditioning stage. Forty pre-exposures and five conditioning trials (strong conditioning) were used. Asterisk indicates a significant difference between the PE and NPE groups, namely, presence of LI.

the mean log times to complete licks 76-100 (after tone onset) of the six experimental groups. As can be seen, LI was absent in vehicle-infused rats and in rats that received scopolamine in the pre-exposure stage, but present in rats that received scopolamine in the conditioning stage. ANOVA yielded a pre-exposure $\times$ intra-BLA treatment interaction $\left(\mathrm{F}_{(2,32)}=3.53, p<0.05\right)$. Post hoc comparisons confirmed presence of LI in rats infused with scopolamine in conditioning $(p<0.02)$, but not in rats infused with vehicle or scopolamine in pre-exposure.

\section{DISCUSSION}

Using two LI protocols, with two or five conditioning trials after 40 stimulus pre-exposures, this study demonstrated a dissociation between the role of muscarinic transmission in the EC and BLA, in the regulation of cognitive processes underlying LI. Vehicle-infused rats showed LI with weak conditioning, but LI was lost with strong conditioning. Intra-EC scopolamine-infused rats failed to show LI with weak conditioning. In contrast, intra-BLA scopolamineinfused rats showed LI with weak and strong conditioning. The effects of intra-BLA and intra-EC scopolamine infusion were further differentiated by the stage at which they acted to produce these LI aberrations. Thus, intra-EC scopolamine abolished LI if infused before pre-exposure or before pre-exposure and conditioning, but not if confined to conditioning. In contrast, intra-BLA scopolamine led to persistent LI if infused before conditioning or before both stages, but not if confined to pre-exposure. These results are consistent with our previous results with systemic scopolamine showing that this drug abolishes LI when injected before pre-exposure (Barak and Weiner, 2007), but induces persistent LI when injected before conditioning (Barak and Weiner, 2009). Our present results suggest that the capacity of systemic scopolamine to induce these contrasting effects on LI may result from its preferred activity at these brain regions during the two stages of the LI procedure. Our results are also consistent with lesion results, showing that EC lesions or its temporary inactivation during pre-exposure abolish LI (Coutureau et al, 1999; Jeanblanc et al, 2004; Lewis and Gould, 2007a; Seillier et al, 2007; Shohamy et al, 2000; Yee et al, 1995), whereas BLA lesions lead to abnormally persistent LI (Schiller and Weiner, 2004, 2005; Schiller et al, 2006; but see Coutureau et al, 2001).

In terms of psychological processes underlying LI, it is believed that during pre-exposure, the acquisition of an association between the PE stimulus and the absence of a significant consequence results in the development of inattention to the stimulus, which inhibits the acquisition and/or the performance of the conditioned response (Bouton, 1993; Lubow and Kaplan, 2005; Weiner, 1990, 2003). Strong conditioning overrides the inhibitory influence of the inattentional response so that animals switch to respond according to the more recent stimulusreinforcement relationship (Weiner, 1990, 2003), leading to loss of LI.

Our finding that intra-EC scopolamine infusion abolishes LI when infused before pre-exposure implies that interference with muscarinic neurotransmission in the EC prevents the acquisition of inattention to irrelevant stimuli, and by extension, that muscarinic transmission in the EC is required for this process. This finding is consistent with other findings and models suggesting a crucial role of cholinergic innervation of the EC in attentional processes in general, and in the acquisition/encoding of associations with novel stimuli in particular (Hasselmo, 2006; Hasselmo and McGaughy, 2004; Hasselmo and Stern, 2006; McGaughy et al, 2005; Ramirez et al, 2007). Notably, although cholinergic innervation of the EC has long been postulated to be involved in the attention to, and encoding of, novel stimuli, our results provide the first evidence that it also has a crucial role in the development of inattention to stimuli. The latter may specifically involve EC-mesolimbic dopamine interactions, as Jeanblanc et al (2004) found that blockade of the EC with tetrodoxin before pre-exposure disrupted normal accumbal DA responses in the $\mathrm{PE}$ condition, in parallel to the disruption of the behavioral expression of LI in these rats.

Scopolamine infusion into the EC before conditioning spared LI, suggesting that muscarinic transmission in this region does not have a role in the expression of the stimulus-no outcome contingency acquired in pre-exposure. Lewis and Gould (2007a) recently reported the same outcome using reversible inactivation of EC by the GABA agonist muscimol in a similar CER procedure. Muscimol infusion during pre-exposure disrupted LI, whereas inactivation during conditioning had no effect. Taken together, these and our results imply that the EC may be the site where the stimulus-no outcome association is formed during pre-exposure, but that this region is not involved in processes occurring in conditioning to both the $\mathrm{PE}$ and the novel stimulus. In addition, Lewis and Gould (2007a) found that inactivation of the EC during the test stage also disrupted LI, suggesting that the EC has a role in both the formation and the retrieval of the stimulus-no outcome contingency acquired in pre-exposure. As the present study has not tested the effects of muscarinic blockade during the test stage, we cannot extend this interpretation to the cholinergic innervation of the EC.

The finding that intra-BLA scopolamine produced persistent LI by action in conditioning implies that 
impaired muscarinic transmission within the BLA does not affect the acquisition of the stimulus-no event association. In fact, animals with impaired muscarinic BLA transmission continue to behave according to the stimulus-no event association under conditions that normally do not support LI. Furthermore, although pharmacological and lesioninduced BLA perturbations, including intra-BLA scopolamine infusion (See et al, 2003), are known to impair conditioning (Cousens and Otto, 1998; Laurent and Westbrook, 2009; Schoenbaum et al, 2003; See et al, 2001), the dose of scopolamine used here did not impair conditioning in the NPE groups conditioned with either two or five trials. It should be noted that there was no behavioral difference between conditioning with two or five trials in the NPE groups suggesting a ceiling effect on performance, but in both cases suppression level was high leaving plenty of room for reduction by intra-BLA muscarinic blockade. In contrast to its lack of effect on conditioning in the NPE groups, intra-BLA scopolamine infusion reduced suppression in the PE group conditioned with five trials. Taken together, these outcomes suggest that muscarinic blockade within the BLA affects conditioning differentially to a PE and a novel stimulus, consistent with our previous results with BLA lesion (Schiller and Weiner 2004). Indeed, as can be seen in Figure 4, intra-BLA scopolamine infusion in conditioning led to the emergence of persistent LI by reducing conditioning in the $\mathrm{PE}$ group, while at the same time having no effect on the NPE group, although both groups had the same level of suppression in the vehicle condition.

The fact that intra-BLA scopolamine infusion impairs performance selectively in the PE group, and that this action is exerted in the conditioning stage, implies that muscarinic blockade in this region specifically impairs the capacity of the PE rats to switch responding from the stimulus-no outcome association acquired in pre-exposure to the stimulus-reinforcement association introduced in conditioning, under conditions triggering such a shift in controls. In other words, although vehicle-infused PE rats conditioned with five trials switch to respond according to the stimulus-reinforcement contingency acquired in conditioning, PE BLA scopolamine-infused rats are apparently unable to adjust their responding to the changed stimulusreinforcement association in conditioning. This outcome is consistent with numerous findings and theories emphasizing the central role of BLA in the capacity to alter responding based upon changes in stimulus-outcomes relationships (Baxter et al, 2000; Corbit and Balleine, 2005; Hatfield et al, 1996; Helms and Mitchell, 2008; Malkova et al, 1997; Schoenbaum et al, 2003), and suggests that muscarinic transmission in the BLA contributes to cognitive flexibility related to outcome significance.

The above explanation is based on the view of LI as a performance/expression deficit resulting from a competition between the stimulus-no event and stimulus-reinforcement associations in conditioning (Weiner 1990, 2003; Bouton 1993). An alternative explanation could be derived from the more traditional attentional models of LI, which view LI as an acquisition deficit, namely, a failure to acquire the CS-reinforcement association as a consequence of the non-reinforced pre-exposure decreasing the attention to, or the associability, of the CS (Lubow, 1989; Lubow and
Kaplan, 2005; Mackintosh, 1975). According to these theories, effective conditioning in normal animals after pre-exposure reflects restored attention to, or associability of, the stimulus, which was reduced by its non-reinforced pre-exposure. Viewed in this light, muscarinic blockade in conditioning can be said to impair the capacity to re-attend to a previously irrelevant stimulus that regains relevance through pairings with reinforcement.

Whatever the precise cognitive mechanisms underlying the recently observed effects, the fact that in rats with impaired muscarinic transmission in the BLA, LI continues to be manifested also when the impact of conditioning increases to a level disrupting LI in normal rats, implies that in the intact brain muscarinic transmission in the BLA is necessary for neither the acquisition nor the expression of LI, but rather for the prevention of its expression (ie, disruption of LI) when the impact of conditioning increases. This stands in marked contrast to the role of the muscarinic transmission of the EC, which is required for the acquisition of LI. It is to be noted that given the anatomical proximity of the BLA to other amygdalar nuclei (ie, the central and lateral nuclei), we cannot completely rule out the possibility that scopolamine diffused to adjacent nuclei. However, as the central nucleus of the amygdala is involved in the expression of fear (LeDoux et al, 1988), and its lesions disrupt fear conditioning (Goosens and Maren, 2001), diffusion to this nucleus would be expected to disrupt fear conditioning; however, we did not observe such a disruption. Although we believe that the effects we observed resulted from muscarinic blockade in the BLA, if there indeed were diffusion to adjacent nuclei, this would imply that not only muscarinic transmission in the BLA but in the entire amygdala is unnecessary for the acquisition/ expression LI, supporting the proposed amygdala-EC dissociation.

Figure 5a depicts a scheme of cholinergic modulation of LI expression, based on a model of brain circuitry regulating LI (Weiner, 1990, 2003), and models of cholinergic-related circuitries mediating attentional processing (Hasselmo and McGaughy, 2004; Sarter et al, 2005; Tzavara et al, 2004; Yeomans, 1995). In the model, LI expression depends on the nucleus accumbens (NAC) and its inputs from the EC, insular cortex (IC), BLA, and prefrontal cortex (PFC). Specifically, switching to respond according to the stimulus-reinforcement contingency (LI abolition) is subserved by increased dopamine (DA) release in the core, which can be inhibited by the shell through its control of DA input to the core from the ventral tegmental area (VTA). The critical input that subserves the inhibition of core switching, namely, the 'CS-no event' information, is relayed to the core by the shell, from the EC and the IC. BLA and PFC relay to the core the information on the motivational value of the CS, which promotes switching to respond according to the CS-reinforcement contingency. Inputs from the PFC and BLA to NAC core, and from the EC and IC to NAC shell (Brog et al, 1993; Chikama et al, 1997; Weiner, 2003; Weiner and Feldon, 1997, Jeanblanc et al, 2004), compete to enhance and reduce, respectively, DA release in the NAC core. BLA, EC, IC, and PFC receive cholinergic afferents from the BF, providing the substrate for cholinergically mediated modulation of these regions' inputs to the NAC. Specifically, muscarinic blockade in the 

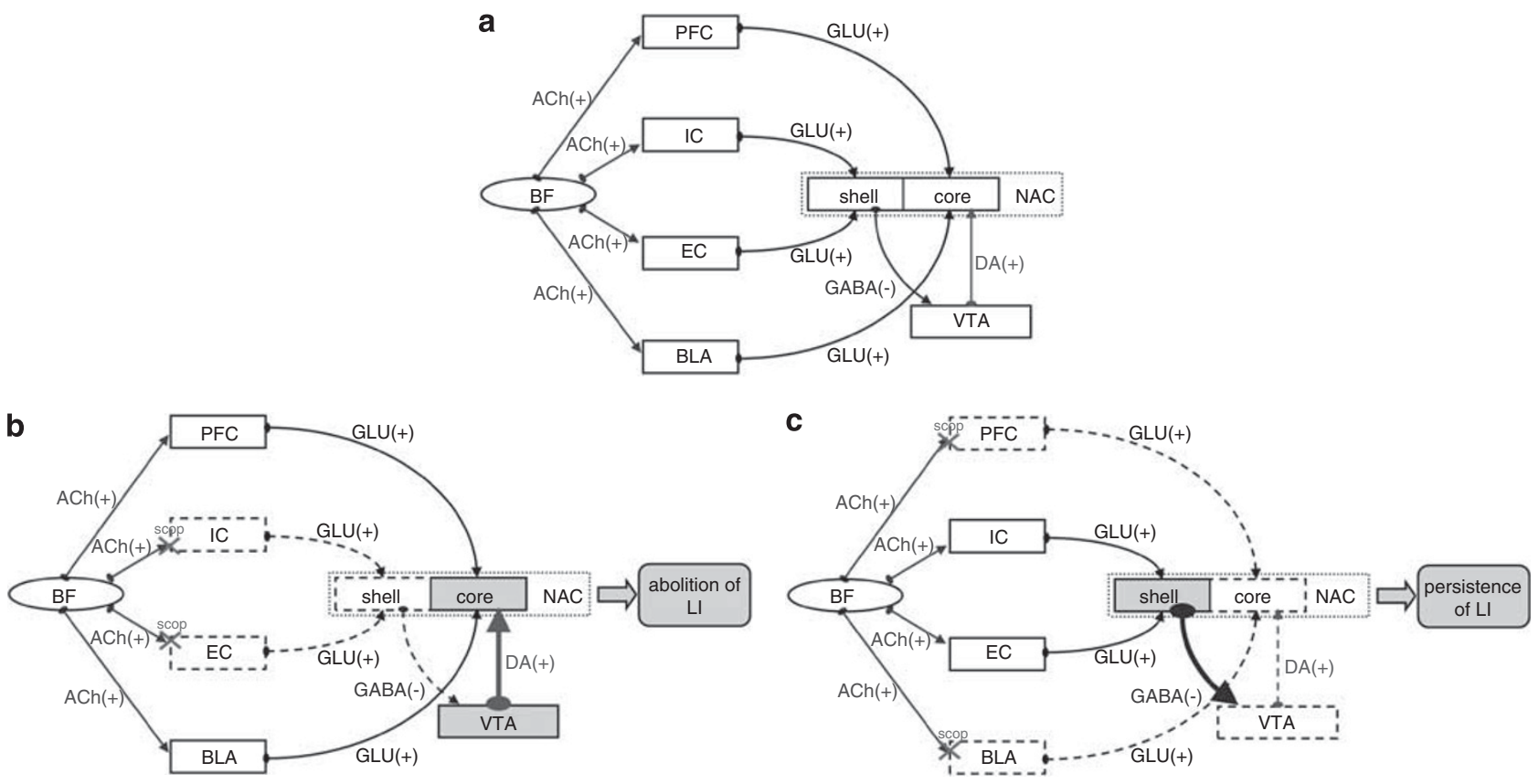

Figure 5 Neural circuitry through which cholinergic projections modulate the expression of latent inhibition. ACh, acetylcholine; BF, basal forebrain; BLA, basolateral amygdala; DA, dopamine; EC, entorhinal cortex; GLU glutamate; IC, insular cortex; LI, latent inhibition; NAC, nucleus accumbens; PFC, prefrontal cortex; SCOP, scopolamine; VTA, ventral tegmental area. (a) The PFC, EC, IC, and BLA receive cholinergic afferents from the BF. Inputs from the PFC and the BLA to the NAC core, and from the EC and IC to the NAC shell enhance and reduce, respectively, DA release from the VTA to the NAC core. Increased and decreased DA levels in the NAC core are associated with LI abolition and persistence, respectively. (b) Muscarinic blockade in the EC or IC inhibits the inputs of these regions to the NAC shell, causing disinhibition of the VTA and enhancing DA release in the NAC core, and leading to abolition of LI. (c) Muscarinic blockade in the BLA inhibits its inputs to the NAC core. Concurrently, the NAC shell, which receives excitatory inputs from the IC and EC, sends inhibitory inputs to the VTA, reducing DA release in the NAC core. Both of these effects lead to LI persistence. Intra-PFC scopolamine infusion is expected to affect LI similarly. This model is based on the switching model of LI (Weiner, 1990, 2003; Weiner and Feldon, 1997), models of cholinergicrelated circuitries mediating attentional processing (Hasselmo and McGaughy, 2004; Sarter et al, 2005; Yeomans, 1995), and LI studies using muscarinic antagonists (Barak, 2009). Figure 5 is adapted from Barak (2009).

EC or IC inhibits the inputs of these regions to the NAC shell, causing disinhibition of the VTA and enhanced DA release in the NAC core, thus leading to LI abolition (Miranda and Bermudez-Rattoni, 2007; Naor and Dudai, 1996). The latter is further facilitated by concurrent excitatory inputs from the PFC and BLA to NAC core (see Figure 5b). Conversely, muscarinic blockade in the BLA inhibits the inputs of this brain region to the NAC core; concurrently, NAC shell, which receives excitatory inputs from the IC and EC, sends inhibitory inputs to the VTA, reducing DA release in the core. Both of these effects lead to LI persistence (see Figure 5c). Intra-PFC scopolamine is expected to affect LI similarly.

This scheme implies that muscarinic transmission in the regions described above mediate attentional processes underlying LI, and by extension, normal attentional processing. Conversely, abnormalities in muscarinic cholinergic transmission in these brain regions may underlie two poles of attentional aberrations manifested in abolished and persistent LI: distractibility caused by impaired ability to in-attend to irrelevant stimuli, mediated by muscarinic transmission in the EC and/or IC; and cognitive rigidity caused by impaired ability re-attend to stimuli that regain relevance, mediated by muscarinic transmission in the BLA and/or the PFC.

Disrupted and persistent LI are considered to model domains of pathology in schizophrenia (Weiner, 2003;
Weiner and Arad, 2009). In particular, scopolamineinduced disrupted LI is considered to model cholinergicrelated positive symptoms (Barak, 2009; Barak and Weiner, 2007; Weiner and Arad, 2009) and scopolamine-induced persistent LI is considered to model antipsychotic drugresistant cognitive impairments of schizophrenia (Barak, 2009; Barak and Weiner, 2009; Weiner and Arad, 2009). Abnormalities of the BLA and the EC have been documented in schizophrenia (Aleman and Kahn, 2005; Arnold, 2000; Benes and Berretta, 2000; Grace, 2000; Haber and Fudge, 1997). Thus, muscarinic transmission in these regions may have a critical role in these abnormalities.

In summary, our results suggest that in the intact brain, muscarinic transmission in the EC and in the BLA have contrasting roles in the regulation of LI expression. Muscarinic transmission in the EC is required for the acquisition of in-attention during pre-exposure, and is therefore essential for the formation of LI. In contrast, muscarinic transmission in the BLA is required for flexible re-attending to stimuli that signal different outcomes than previously experienced, and is therefore essential for LI abolition. These findings suggest that abnormalities in muscarinic cholinergic transmission in these brain regions may have a key role in the cognitive lability and cognitive rigidity observed in schizophrenia and other neuropsychiatric disorders. 


\section{ACKNOWLEDGEMENTS}

This research was supported by the Israel Science Foundation (Grant No. 1234/07) and by the Josef Sagol Fellowship Program in Brain Studies at Tel-Aviv University (SB).

\section{DISCLOSURE}

The authors declare no conflict of interest.

\section{REFERENCES}

Aleman A, Kahn RS (2005). Strange feelings: do amygdala abnormalities dysregulate the emotional brain in schizophrenia? Prog Neurobiol 77: 283-298.

Arnold SE (2000). Cellular and molecular neuropathology of the parahippocampal region in schizophrenia. Ann NY Acad Sci 911: 275-292.

Barak S (2009). Modeling cholinergic aspects of schizophrenia: Focus on the antimuscarinic syndrome. Behav Brain Res 204: 335-351.

Barak S, Weiner I (2007). Scopolamine induces disruption of latent inhibition which is prevented by antipsychotic drugs and an acetylcholinesterase inhibitor. Neuropsychopharmacology 32: 989-999.

Barak S, Weiner I (2009). Towards an animal model of an antipsychotic drug-resistant cognitive impairment in schizophrenia: scopolamine induces abnormally persistent latent inhibition, which can be reversed by cognitive enhancers but not by antipsychotic drugs. Inter J Neuropsychopharmacol 12: 227-241.

Barros DM, Mello e Souza T, De David T, Choi H, Aguzzoli A, Madche $C$ et al (2001). Simultaneous modulation of retrieval by dopaminergic $\mathrm{D}(1)$, beta-noradrenergic, serotonergic-1A and cholinergic muscarinic receptors in cortical structures of the rat. Behav Brain Res 124: 1-7.

Baxter MG, Parker A, Lindner CC, Izquierdo AD, Murray EA (2000). Control of response selection by reinforcer value requires interaction of amygdala and orbital prefrontal cortex. J Neurosci 20: 4311-4319.

Benes FM, Berretta S (2000). Amygdalo-entorhinal inputs to the hippocampal formation in relation to schizophrenia. Ann $N Y$ Acad Sci 911: 293-304.

Blokland A (1995). Acetylcholine: a neurotransmitter for learning and memory? Brain Res Rev 21: 285-300.

Bouton ME (1993). Context, time, and memory retrieval in the interference paradigms of Pavlovian learning. Psychol Bull 114: 80-99.

Brog JS, Salyapongse A, Deutch AY, Zahm DS (1993). The patterns of afferent innervation of the core and shell in the 'accumbens' part of the rat ventral striatum: immunohistochemical detection of retrogradely transported fluoro-gold. J Comp Neurol 338: 255-278.

Chhatwal JP, Gutman AR, Maguschak KA, Bowser ME, Yang Y, Davis $M$ et al (2009). Functional interactions between endocannabinoid and CCK neurotransmitter systems may be critical for extinction learning. Neuropsychopharmacology 34: 509-521.

Chikama M, McFarland NR, Amaral DG, Haber SN (1997). Insular cortical projections to functional regions of the striatum correlate with cortical cytoarchitectonic organization in the primate. J Neurosci 17: 9686-9705.

Corbit LH, Balleine BW (2005). Double dissociation of basolateral and central amygdala lesions on the general and outcome-specific forms of pavlovian-instrumental transfer. $J$ Neurosci 25: 962-970.

Cousens G, Otto T (1998). Both pre- and posttraining excitotoxic lesions of the basolateral amygdala abolish the expression of olfactory and contextual fear conditioning. Behav Neurosci 112: 1092-1103.

Coutureau E, Blundell PJ, Killcross S (2001). Basolateral amygdala lesions disrupt latent inhibitionin rats. Brain Res Bull 56: 49-53. Coutureau E, Galani R, Gosselin O, Majchrzak M, Di Scala G (1999). Entorhinal but not hippocampal or subicular lesions disrupt latent inhibition in rats. Neurobiol Learn Mem 72: 143-157.

Coutureau E, Gosselin O, Di Scala G (2000). Restoration of latent inhibition by olanzapine but not haloperidol in entorhinal cortex-lesioned rats. Psychopharmacology (Berl) 150: 226-232.

Ebert U, Kirch W (1998). Scopolamine model of dementia: electroencephalogram findings and cognitive performance. Eur J Clin Invest 28: 944-949.

Everitt BJ, Robbins TW (1997). Central cholinergic systems and cognition. Annu Rev Psychol 48: 649-684.

Fibiger HC (1991). Cholinergic mechanisms in learning, memory and dementia: a review of recent evidence. Trends Neurosci 14: 220-223.

Goosens KA, Maren S (2001). Contextual and auditory fear conditioning are mediated by the lateral, basal, and central amygdaloid nuclei in rats. Learn Mem 8: 148-155.

Grace AA (2000). Gating of information flow within the limbic system and the pathophysiology of schizophrenia. Brain Res Brain Res Rev 31: 330-341.

Green A, Ellis KA, Ellis J, Bartholomeusz CF, Ilic S, Croft RJ et al (2005). Muscarinic and nicotinic receptor modulation of object and spatial n-back working memory in humans. Pharmacol Biochem Behav 81: 575-584.

Haber SN, Fudge JL (1997). The interface between dopamine neurons and the amygdala: implications for schizophrenia. Schizophr Bull 23: 471-482.

Hasselmo ME (2006). The role of acetylcholine in learning and memory. Curr Opin Neurobiol 16: 710-715.

Hasselmo ME, McGaughy J (2004). High acetylcholine levels set circuit dynamics for attention and encoding and low acetylcholine levels set dynamics for consolidation. Prog Brain Res 145: 207-231.

Hasselmo ME, Stern CE (2006). Mechanisms underlying working memory for novel information. Trends Cogn Sci 10: 487-493.

Hatfield T, Han JS, Conley M, Gallagher M, Holland P (1996). Neurotoxic lesions of basolateral, but not central, amygdala interfere with Pavlovian second-order conditioning and reinforcer devaluation effects. J Neurosci 16: 5256-5265.

Helms CM, Mitchell SH (2008). Basolateral amygdala lesions and sensitivity to reinforcer magnitude in concurrent chains schedules. Behav Brain Res 191: 210-218.

Herremans AH, Hijzen TH, Welborn PF, Olivier B, Slangen JL (1996). Effects of infusion of cholinergic drugs into the prefrontal cortex area on delayed matching to position performance in the rat. Brain Res 711: 102-111.

Ingles JL, Beninger RJ, Jhamandas K, Boegman RJ (1993). Scopolamine injected into the rat amygdala impairs working memory in the double Y-maze. Brain Res Bull 32: 339-344.

Jeanblanc J, Peterschmitt Y, Hoeltzel A, Louilot A (2004). Influence of the entorhinal cortex on accumbal and striatal dopaminergic responses in a latent inhibition paradigm. Neuroscience 128: $187-200$

Laurent V, Westbrook RF (2009). Infusion of the NMDA receptor antagonist, DL-APV, into the basolateral amygdala disrupts learning to fear a novel and a familiar context as well as relearning to fear an extinguished context. Learn Mem 16: 96-105.

LeDoux JE, Iwata J, Cicchetti P, Reis DJ (1988). Different projections of the central amygdaloid nucleus mediate autonomic and behavioral correlates of conditioned fear. J Neurosci 8: 2517-2529.

Lewis MC, Gould TJ (2007a). Reversible inactivation of the entorhinal cortex disrupts the establishment and expression of 
latent inhibition of cued fear conditioning in C57BL/6 mice. Hippocampus 17: 462-470.

Lewis MC, Gould TJ (2007b). Signal transduction mechanisms within the entorhinal cortex that support latent inhibition of cued fear conditioning. Neurobiol Learn Mem 88: 359-368.

Lubow RE (1989). Latent Inhibition and Conditioned Attention Theory. Cambridge University Press: New York.

Lubow RE, Kaplan O (2005). The visual search analogue of latent inhibition: implications for theories of irrelevant stimulus processing in normal and schizophrenic groups. Psychon Bull Rev 12: 224-243.

Lubow RE, Weiner I (eds) (2009). Latent Inhibition: Data, Theories, and Applications to Schizophrenia. Cambridge University Press: New York.

Mackintosh NJ (1975). A theory of attention: variations in the associability of stimuli with reinforcement. Psychol Rev 82: 276-298.

Malkova L, Gaffan D, Murray EA (1997). Excitotoxic lesions of the amygdala fail to produce impairment in visual learning for auditory secondary reinforcement but interfere with reinforcer devaluation effects in rhesus monkeys. J Neurosci 17: 6011-6020.

McGaughy J, Koene RA, Eichenbaum H, Hasselmo ME (2005). Cholinergic deafferentation of the entorhinal cortex in rats impairs encoding of novel but not familiar stimuli in a delayed nonmatch-to-sample task. J Neurosci 25: 10273-10281.

Milton AL, Lee JL, Butler VJ, Gardner R, Everitt BJ (2008). Intraamygdala and systemic antagonism of NMDA receptors prevents the reconsolidation of drug-associated memory and impairs subsequently both novel and previously acquired drug-seeking behaviors. J Neurosci 28: 8230-8237.

Miranda MI, Bermudez-Rattoni F (2007). Cholinergic activity in the insular cortex is necessary for acquisition and consolidation of contextual memory. Neurobiol Learn Mem 87: 343-351.

Naor C, Dudai Y (1996). Transient impairment of cholinergic function in the rat insular cortex disrupts the encoding of taste in conditioned taste aversion. Behav Brain Res 79: 61-67.

Paxinos G, Watson C (1998). The Rat Brain in Stereotaxic Coordinates, 4th edn. Academic Press: San Diego.

Power AE (2004). Muscarinic cholinergic contribution to memory consolidation: with attention to involvement of the basolateral amygdala. Curr Med Chem 11: 987-996.

Power AE, Vazdarjanova A, McGaugh JL (2003). Muscarinic cholinergic influences in memory consolidation. Neurobiol Learn Mem 80: 178-193.

Ramirez JJ, Campbell D, Poulton W, Barton C, Swails J, Geghman K et al (2007). Bilateral entorhinal cortex lesions impair acquisition of delayed spatial alternation in rats. Neurobiol Learn Mem 87: 264-268.

Roesler R, Roozendaal B, McGaugh JL (2002). Basolateral amygdala lesions block the memory-enhancing effect of 8-BrcAMP infused into the entorhinal cortex of rats after training. Eur J Neurosci 15: 905-910.

Sarter M, Bruno JP, Givens B (2003). Attentional functions of cortical cholinergic inputs: what does it mean for learning and memory? Neurobiol Learn Mem 80: 245-256.

Sarter M, Nelson CL, Bruno JP (2005). Cortical cholinergic transmission and cortical information processing in schizophrenia. Schizophr Bull 31: 117-138.

Schauz C, Koch M (2000). Blockade of NMDA receptors in the amygdala prevents latent inhibition of fear-conditioning. Learn Mem 7: 393-399.

Schiller D, Weiner I (2004). Lesions to the basolateral amygdala and the orbitofrontal cortex but not to the medial prefrontal cortex produce an abnormally persistent latent inhibition in rats Neuroscience 128: 15-25.

Schiller D, Weiner I (2005). Basolateral amygdala lesions in the rat produce an abnormally persistent latent inhibition with weak preexposure but not with context shift. Behav Brain Res 163: 115-121.

Schiller D, Zuckerman L, Weiner I (2006). Abnormally persistent latent inhibition induced by lesions to the nucleus accumbens core, basolateral amygdala and orbitofrontal cortex is reversed by clozapine but not by haloperidol. J Psychiatr Res 40: 167-177.

Schoenbaum G, Setlow B, Nugent SL, Saddoris MP, Gallagher M (2003). Lesions of orbitofrontal cortex and basolateral amygdala complex disrupt acquisition of odor-guided discriminations and reversals. Learn Mem 10: 129-140.

See RE, Kruzich PJ, Grimm JW (2001). Dopamine, but not glutamate, receptor blockade in the basolateral amygdala attenuates conditioned reward in a rat model of relapse to cocaine-seeking behavior. Psychopharmacology (Berl) 154: 301-310.

See RE, McLaughlin J, Fuchs RA (2003). Muscarinic receptor antagonism in the basolateral amygdala blocks acquisition of cocaine-stimulus association in a model of relapse to cocaineseeking behavior in rats. Neuroscience 117: 477-483.

Seillier A, Dieu Y, Herbeaux K, Di Scala G, Will B, Majchrzak M (2007). Evidence for a critical role of entorhinal cortex at preexposure for latent inhibition disruption in rats. Hippocampus 17: $220-226$.

Shohamy D, Allen MT, Gluck MA (2000). Dissociating entorhinal and hippocampal involvement in latent inhibition. Behav Neurosci 114: 867-874.

Tzavara ET, Bymaster FP, Davis RJ, Wade MR, Perry KW, Wess J et al (2004). M4 muscarinic receptors regulate the dynamics of cholinergic and dopaminergic neurotransmission: relevance to the pathophysiology and treatment of related CNS pathologies. FASEB J 18: 1410-1412.

Warburton EC, Koder T, Cho K, Massey PV, Duguid G, Barker GR et al (2003). Cholinergic neurotransmission is essential for perirhinal cortical plasticity and recognition memory. Neuron 38: 987-996.

Weiner I (1990). Neural substrates of latent inhibition: the switching model. Psychol Bull 108: 442-461.

Weiner I (2003). The 'two-headed' latent inhibition model of schizophrenia: modeling positive and negative symptoms and their treatment. Psychopharmacology (Berl) 169: 257-297.

Weiner I, Arad M (2009). Using the pharmacology of latent inhibition to model domains of pathology in schizophrenia and their treatment. Behav Brain Res 204: 369-386.

Weiner I, Feldon J (1997). The switching model of latent inhibition: an update of neural substrates. Behav Brain Res 88: 11-25.

Winters BD, Saksida LM, Bussey TJ (2006). Paradoxical facilitation of object recognition memory after infusion of scopolamine into perirhinal cortex: implications for cholinergic system function. J Neurosci 26: 9520-9529.

Yee BK, Feldon J, Rawlins JN (1995). Latent inhibition in rats is abolished by NMDA-induced neuronal loss in the retrohippocampal region, but this lesion effect can be prevented by systemic haloperidol treatment. Behav Neurosci 109: 227-240.

Yeomans JS (1995). Role of tegmental cholinergic neurons in dopaminergic activation, antimuscarinic psychosis and schizophrenia. Neuropsychopharmacology 12: 3-16.

Zhao Z, Yang Y, Walker DL, Davis M (2009). Effects of substance P in the amygdala, ventromedial hypothalamus, and periaqueductal gray on fear-potentiated startle. Neuropsychopharmacology 34: $331-340$

Supplementary Information accompanies the paper on the Neuropsychopharmacology website (http://www.nature.com/npp) 